\title{
Cirugía Centrada en la Persona
}

\author{
Person centered surgery \\ Daniel Enrique Haro-Haro' ${ }^{2}$ Augusto B. Chafloque-Cervantes ${ }^{2}$
}

\section{Resumen}

Este trabajo tiene como objetivo hacer una revisión de la cirugía centrada en la persona, los antecedentes históricos, los principios básicos del ejercicio de la profesión y el perfil del cirujano humanista y humanitario, priorizando el vínculo con el paciente y las metas que se debe alcanzar en los planes curriculares de su formación, y enfatizando en la importancia del consentimiento informado en cirugía. Los autores hacen una propuesta para mejorar su eficacia, haciendo énfasis en que, en todos momento, el cirujano debe aceptar con honestidad y ética requisitos indispensables para una buena práctica profesional, brindando su capacidad en beneficio del paciente.

Palabras clave: Cirugía, persona, ética, humanismo, humanitarismo.

\section{Abstract}

This work aims to make a review of the person-centered surgery, the historical background, the basic principles of the surgical practice, and the profile of the humanist and humanitarian surgeon. It prioritizes the bond with the patient and the goals to be achieved in the curricular plans of surgeon training, and analyzed the importance of informed consent in surgery. The authors make a proposal to improve its effectiveness, emphasizing that, at all times, surgeons must accept with honesty and ethics, essential requirements for a good professional practice, offering his ability for the benefit of the patient.

Keywords: Surgery, person, ethic, humanism, humanitarianism.

\section{Introducción}

La medicina centrada en la persona es importante, pero mucho más trascendente es la cirugía centrada en la persona. A diferencia de la primera, la cirugía conlleva en el acto quirúrgico una invasión en el cuerpo del paciente y ello lo relaciona más íntimamente con los principios rectores de la ética y deontología médica, convirtiendo al paciente en el centro de atención mas allá del tipo de padecimiento.

Siendo la cirugía en esencia una profesión humanista y de servicio, se considera una obligación moral que esté centrada en la persona. En este sentido, la actitud del cirujano frente a un paciente debe considerar siempre los aspectos biológico o corporal, psicológico y socio-cultural. El humanismo gira necesariamente alrededor de una adecuada relación médico-paciente, en la que el cirujano considera a su paciente como un ser humano, como una persona, y le brinda sus conocimientos, destreza, habilidad y experiencia con respeto y consideración. Solo así el paciente tendrá la confianza necesaria para someterse a una intervención quirúrgica.

La visión humanista del cirujano requiere, además, bondad y comprensión, valorando al ser humano en su condición de enfermo, que entrega su salud y su vida a quien con sus manos va a realizar un acto quirúrgico para mejorar y/o curar su salud y, a veces, salvar su vida. En este contexto, el cirujano se debe acercar al hombre en su esencia. Decía Gregorio Marañón: "Solo hay un camino para llegar a la ciencia quirúrgica: el aprendizaje y el adiestramiento. Y hay otro camino para llegar al humanismo y al humanitarismo: el espíritu templado y generoso, ${ }^{(1,2)}$.

${ }^{1}$ Doctor en Medicina, Oftalmólogo, Ex-Profesor Principal de la Universidad Peruana Cayetano Heredia, Ex-Presidente de la Sociedad Peruana de Oftalmología y de la Academia Peruana de Cirugía, Ex Jefe del Sevicio de Oftalmología del Hospital A. Loayza.

${ }^{2}$ Doctor en Medicina, Oftalmólogo, Profesor Princiipal y Jefe de la Unidad de Post grado de Oftalmología, Facultad de Medicina. Universidad Nacional Mayor de San Marcos, Jefe del Servicio de Oftalmología del Instituto Nacional Materno Perinatal. 
El humanismo en el cirujano lo hace más fuerte y culto, le ayuda a entender más al enfermo con sus aspiraciones, temores y miserias. Le enseña a separar lo útil de lo inútil, lo bueno de lo malo y lo justo de lo injusto; le obliga a contraer su responsabilidad a plenitud con sentido de integridad, equidad y ética, dejando de lado factores raciales, ideológicos, de género, religiosos, económicos y culturales ${ }^{(3)}$.

Todo médico-cirujano debe ser humanitario, es decir, generoso y compasivo, y debe preocuparse por valorar los derechos fundamentales de la persona. Sobre todo del paciente asertivo con relación a la vida y a la salud. Debe tratar de que se realicen sus deseos, aspiraciones y expectativas, respetando su negativa ante alguna propuesta que no le parezca conveniente. La cirugía centrada en la persona obliga al cirujano a brindar la mejor atención y cuidado del paciente en base a los siguientes principios rectores $^{(4,5)}$ :

-Beneficencia y no maleficiencia, cumpliendo lo estipulado en el Juramento Hipocrático, "primo non nocere”, y siempre hacer el bien respetando su dignidad.

-Autonomía y libertad, que involucra al ser humano como un sujeto y no como un objeto, con capacidad de elegir sin coacción lo que considere mejor para sí mismo, sin intentar convertirse en autosuficiente.

-Justicia y equidad, que obliga a ofrecer el mejor servicio con calidad y calidez, y sin discriminaciones.

-Solidaridad, entendimiento y responsabilidad, que es el compromiso afectivo con el paciente y siempre con trato respetuoso.

-Veracidad, actuando siempre con la verdad en relación al tratamiento quirúrgico, sin ocultar los riesgos de la intervención.

La situación del cirujano en relación al paciente que requiere una cirugía ha sufrido cambios profundos en estos últimos años debido a varios factores, que incluyen:

-La globalización y el gran desarrollo de la informática, que hace fácil encontrar en internet todo lo relacionado con la enfermedad del paciente.

-La promoción de la autonomía del paciente y el mejor conocimiento de sus derechos, que incluye al consentimiento informado.

-La socialización de la medicina y los seguros de salud con sus exigencias, que deteriora la relación médico-paciente, limitando el trabajo del cirujano e impidiendo una adecuada atención $^{(6,7)}$.

En las secciones siguientes haremos la revisión de la historia del tema que nos ocupa, la trascendencia del humanismo y humanitarismo, la influencia de la ética y bioética en la formación del cirujano y la importancia del consentimiento informado, teniendo en cuenta la atención quirúrgica multidisciplinaria para tomar decisiones compartidas por todos los involucrados, pero siempre centrada en la persona.

\section{Historia}

Vivimos cada vez más en una cultura del instante, en la que solo existe el día a día, en la que no hay raíces. Campea el olvido y la desmemoria y, sin embargo, la realidad es otra, todo en la vida tiene raíces sin las cuales es imposible ubicarse. Estas raíces son la memoria, es decir, la historia. Por ello, en esta reseña pretendemos mostrar la historia de la medicina y cirugía centrada en la persona.

Al principio de los tiempos, el ser humano solo podía explicar su existencia mediante los mitos, de naturaleza mágica y llenos de concepciones que hoy denominamos superticiones. En la mitología griega, Asklepsios es el Dios de la medicina. Su nombre significa "incesantemente benévolo". Según relatan antiguos escritos, Asklepsios era tan bondadoso que no solo curaba a los enfermos sino que, contrariando a las leyes de su padre, Zeus, se atrevió a resucitar muertos. Por esa osadía el autor de sus días le quitó la vida. Este hecho encierra una lección que demuestra que, incluso en los mitos, ningún médico puede crear la vida ni evitar la muerte.

La palabra médico deriva de "Medum" en griego y "medicum" en latín y equivale a la persona que hace el bien, cura, consuela y aconseja. Esto es más cierto en el significado del cirujano, que deriva de la palabra "cirugía", del griego "kheiros" (manos) y "ergon" (obra). Ha sido definida como aquella parte de la medicina que trata de curar las enfermedades por obra de las manos.

En tiempos remotos, en Babilonia (2000 a.c.), existía el Código de Hammurabi que representó el primer intento legislativo del ser humano. De las 282 leyes que contiene, algunas se refieren al ejercicio de la medicina y la cirugía.

En Mesopotamia, (1000 a.c), se reguló un Código de Ética para la práctica de la cirugía y las sanciones, algunas muy drásticas, como cortarle la mano al cirujano cuando se perdía el ojo de algún personaje importante como consecuencia de una mala operación de catarata. 
De Esculapio e Hipócrates hemos aprendido que el comportamiento ético es el espíritu que en la historia de la humanidad ha animado la práctica médica y quirúrgica. Su incorporación al ejercicio de nuestra profesión tiene una larga tradición, que se inicia en Grecia con el "Juramento Hipocrático" considerado como el referente ético en el mundo occidental. Hipócrates, médico griego (460 a.c.), creó el Juramento que resume los principios éticos que guían al médico-cirujano en su labor cotidiana y regula las obligaciones hacia sus maestros, discípulos, parientes y colegas $^{(8,9)}$.

El Juramento Hipocrático ha tenido varios intentos de modificaciones en el transcurso del tiempo. A la primera actualización de dicho Juramento, realizada por la Asociación Médica Mundial, se le conoce como "Declaración de Ginebra" en el año 1948. Actualmente, es vigente la adoptada por la Asociación Médica Mundial de Chicago en 2017, que se denomina el "Juramento del Médico" y representa la consagración del médico a su profesión y contiene el siguiente texto $^{(10-12)}$ :

\section{Prometo:}

- Consagrar mi vida al servicio de la humanidad.

- Otorgar a mis maestros el respeto y la gratitud que merecen.

- Ejercer mi profesión dignamente y a conciencia.

- Velar por la salud de mis pacientes.

- Guardar y respetar el secreto profesional.

- Mantener incólume el honor y las nobles tradiciones de la profesión médica.

- Considerar como hermanos a mis colegas.

- Hacer caso omiso a credos políticos y religiosos, nacionalidades, razas, rangos sociales y económicos, evitando que se interpongan entre mis servicios profesionales y mi paciente.

- Mantener sumo respeto por la vida humana desde el momento mismo de la concepción y no utilizar mis conocimientos para contravenir las leyes de la humanidad.

En el Nuevo Testamento, Lucas (Lc.10.27-37), médico a quien se le denomina "El Evangelista de la Misericordia", nos presenta en la "Parábola del Buen Samaritano" el significado de la deontología médica inspirada en el espíritu de caridad y solidaridad compasiva de los cristianos, y nos recuerda aquello que es como un mandamiento: "Ama a tu prójimo como a ti mismo" ${ }^{(13)}$.

La evolución histórica de la cirugía, que antaño la realizaban los barberos, ha mostrado cambios radicales y matices extraordinarios, desde su marginación y rechazo a nivel universitario, al no reconocerla como ciencia médica, hasta su ascenso imparable a partir del siglo XIX. Dicha evolución la ha llevado a niveles insospechados de especialización que, lamentablemente, deshumanizan al cirujano y lo alejan del paciente.

Ambroise Paré (1510-1590), principal figura quirúrgica del siglo XVI a cuya ejemplar vida no se le ha dado la importancia y trascendencia debidas, no solo estableció las bases de la cirugía moderna, sino que también se distinguió por su humildad en una época de tanta crueldad. Paré rompió la ética elitista para ayudar al necesitado sin hacer distinción alguna, dándole sentido moral a su obra. Consideramos por ello que fue el primer cirujano en la historia mundial en practicar la cirugía centrada en la persona. Dejó de ser un simple barbero para convertirse en médico y cirujano de reyes. Considerado el padre de la cirugía, a él se atribuye la siguiente afirmación: “ $L a$ cirugía tiene como funciones eliminar lo superfluo, restaurar lo que se ha dislocado, separar lo que se ha unido, reunir lo que se ha dividido y reparar los defectos de la naturaleza”. Esta frase sigue teniendo vigencia en la actualidad y debemos cumplirla a cabalidad $^{(14)}$.

En 1543, el Royal College of Physicians de Inglaterra emitió las normas regulatorias para el ejercicio de la medicina y la cirugía. En 1628, hicieron lo mismo en el Protomedicato de Nueva España. En el año 1770 apareció la obra de John Gregory "Observations on the Duties and Offices of a Physician and on the Method of Prosecuting Enquiries in Phylosophy", The Code of Medical Ethics de Thomas Percival en 1806 y el Código de Ética de la American Medical Association en 1847.

En 1913 se fundó el Colegio Americano de Cirujanos, institución en la cual se establecieron los principios éticos para los cirujanos y se dictaron normas y altos estándares para la práctica quirúrgica y para la educación especializada. Desde entonces, sus colegiados hacen "la promesa de procurar el bienestar de los pacientes por encima de todo". Posteriormente, en el año 1994 publicaron un folleto en el que recopilaron las "Normas para el cuidado de los pacientes, los deberes y obligaciones del cirujano". Todo ello como respuesta al surgimiento de la bioética, los grandes avances tecnológicos, las presiones económicas y el respeto de los derechos de los pacientes $^{(14)}$.

Respecto al profesionalismo en el nuevo milenio, la American Board of Internal Medicine (ABIM), el American College of Physicians (ACP), la American Society of Internal Medicine (ASIM) y la European Federation of Internal Medicine (EFIM) contemplan un código en el que se señala 
como preámbulo que "el profesionalismo es la base del contrato de la medicina y cirugía con la sociedad". Ello exige que debamos colocar los intereses del paciente por encima de los nuestros, estableciendo niveles de competencia e integridad ${ }^{(15)}$.

Si la definición de la salud es el estado de completo bienestar físico, psíquico y social y depende de las condiciones socio-económicas, en países pobres como el nuestro, en los que existe tanta desigualdad, la salud no alcanza a las grandes mayorías. Por lo tanto, no existe la medicina y cirugía centrada en la persona, ni siquiera en el primer nivel de atención primaria.

Para Diego Gracia, "el genuino profesionalismo implica tres elementos esenciales: la devoción o sea el compromiso de entrega altruista, el reconocimiento de la sociedad del valor de la profesión médica a la que le confiere un elevado status y el compromiso social". Por este compromiso los médicos cirujanos nos imponemos el deber de defender el valor de la persona humana, respetando el derecho del paciente de recibir información precisa y clara y de participar en la toma de decisiones en lo que respecta a su salud y su vida ${ }^{(16)}$.

La Declaración de Alma-Ata en Kasajistán en el año de 1978 definió la atención primaria de salud como un conjunto de valores que ayudan al desarrollo sanitario de los pueblos, resaltando la importancia de la igualdad socio-económica y especialmente en salud en países en desarrollo. En esa importante reunión David Tejada de Rivero fue Coordinador General de la Conferencia y artífice de sus conclusiones. Él nos enseñó que mejorar la calidad de vida es dar buena salud y con ello obtener los logros y la realización de las aspiraciones personales, familiares y de la sociedad en su conjunto ${ }^{(17)}$.

En el Perú Republicano, la cirugía tiene como institución rectora a la Academia Peruana de Cirugía, cuyo origen se remonta a la fundación de la Academia Nacional de Medicina en el año 1888, institución que llegó a convertirse en el más alto templo de la cultura médica y quirúrgica en la vida de nuestra nación, siendo su primer presidente José María Romero eminente cirujano. Hasta el año 1914 las Directivas eran de alternancia cada dos años entre un médico y un cirujano. En ese año, devino la separación de los cirujanos y la constitución de la Sociedad Peruana de Cirugía que luego, en el año 1940, cambió su denominación por la actual y que, hasta hoy, congrega a lo más selecto de los cirujanos de nuestro país ${ }^{(18)}$.

En el siglo pasado los grandes maestros de la cirugía peruana, como Constantino Carvallo, Guillermo Gastañeta y otros ilustres cirujanos, consiguieron notables avances. Fruto de su audacia, trasformaron el arte operatorio en ciencia quirúrgica, constituyéndose las diversas especialidades quirúrgicas que existen hoy en día y que se han enriquecido grandemente con la microcirugía, los rayos láser, la cirugía laparoscópica y robótica, lo que nos obliga a actualizar nuestros conocimientos, habilidades y destrezas, involucrándonos con el mundo de la tecnología moderna, la investigación y la nueva normatividad ${ }^{(19)}$.

En sus 80 años de vigencia, la Academia Peruana de Cirugía ha mantenido el prestigio institucional y su misión ha sido siempre la docencia, difundiendo los nuevos conocimientos y adelantos, promoviendo el progreso de las especialidades quirúrgicas y sobre todo la vigilancia de la ética y deontología profesional $^{(20)}$.

\section{Humanismo y Humanitarismo}

El Diccionario de la Real Academia Española, en una de sus definiciones del Humanismo dicta: "El humanismo es la doctrina o actitud vital basada en una concepción integradora de los valores humanos". En medicina y cirugía esto quiere decir "centrar laatención en la persona",(21).

Ser cirujano humanista es ser culto, comprender al hombre con sus aspiraciones y miserias, valorar lo bueno, lo bello y lo justo de la vida. En tal sentido, han surgido en varios países movimientos para priorizar a la persona como centro de la salud, cuyas raíces tempranas fueron encontradas en las antiguas civilizaciones de China y $\mathrm{Grecia}^{(22,23)}$.

En el Perú, siguiendo el ejemplo de Honorio Delgado ${ }^{(24)}$ y Carlos Alberto Seguín ${ }^{(25)}$, se están realizando esfuerzos para conseguir dicho fin. La atención centrada en la persona ha sido una aspiración histórica de la medicina y la cirugía, a pesar de que últimamente ésta se ha ido perdiendo por diversos motivos ligados a la focalización de la enfermedad y sus órganos afectados. Esta creciente especialización conduce a la fragmentación de la cirugía ${ }^{(26,27)}$.

Siguiendo las normas establecidas, el desempeño del cirujano se basa en el humanismo, la prudencia, la solidaridad, la confidencialidad y la honestidad ${ }^{(28,29)}$ :

-El humanismo, que implica una visión y conocimiento amplio, y vasta cultura. Recordemos la sentencia de José de Letamendi: "El médico que solo sabe medicina, ni medicina sabe".

-La prudencia o frónesis, que significa inteligencia serena, sensatez y actitud prudente. Lo opuesto a ello es la 
frénesis o imprudencia, que es la actitud frenética que todo lo hace apresuradamente. Esto es temerario en cirugía.

-La solidaridad, que significa identificación, adhesión y apoyo, en la que "el cirujano se pone en el lugar del enfermo que sufre" y centra su labor en la persona que atiende.

-La confidencialidad, que incluye el secreto profesional, tan importante en el diario quehacer del cirujano.

-La honestidad y decoro, porque si no hay honradez en el desempeño profesional, éste se invalida de raíz.

La cirugía centrada en la persona obliga a todos los cirujanos a amar al ser humano, obrar desinteresadamente, detentar bondad y compresión, tener valores, principios y normas de conducta bien cimentados, sensibilidad humana con el paciente y respeto a sus derechos, poseer vocación de servicio, sobre todo con los más necesitados, conseguir sabiduría y sólidos conocimientos en su formación profesional, adquirir habilidad, destreza y experiencia en su especialidad, balancear los avances tecnológicos con el humanismo, e identificarse con el paciente, poniéndose en su lugar.

Consistentemente, las características del proceso formativo del residente de cirugía, en cualquier especialidad, deben incluir comportamiento idóneo, adquisición de habilidades y destrezas, programas docentes actualizados y centros quirúrgicos bien equipados. Solo así se tendrá una formación integral. "El médico-cirujano solo ve lo que sabe y lo que no se aprende no se puede ejercer" ${ }^{(30,31)}$.

En la educación y formación del cirujano, además de la ciencia quirúrgica, se debe enseñar el cultivo de la sensibilidad humana y el amor a los pacientes, procurando desarrollar una actitud solidaria y el respeto a sus derechos. Se le debe exhortar a tener calidad y calidez en la atención a los pacientes, respetando su dignidad, y recordando la antigua sentencia que reza: "Trata a tu paciente como quisieras que te traten a ti mismo".

Además, se debe enseñar que en todo proceso quirúrgico deben cumplirse los siguientes pasos para asegurar el éxito de la cirugía: estudio minucioso de la historia clínica, analizando los síntomas y signos; los exámenes auxiliares; revisión del consentimiento informado debidamente firmado; un adecuado tratamiento pre-quirúrgico; $\mathrm{y}$, sobre todo, practicar la cirugía segura y un seguimiento minucioso en el postoperatorio $^{(32,33)}$.

Santa Teresa de Calcuta (1919-1997) expresó: "Si no se vive para los demás, la vida no tiene sentido” ¡Gran verdad para los médicos y cirujanos!. En una visita al Perú, dijo a ellos: "Sean caritativos con las personas que atienden y tengan en cuenta que los pacientes siempre están en un estado de indefensión frente a la enfermedad".

Las razones que alejan a los cirujanos de la cirugía centrada en la persona pueden ser de índole personal, por causa de la indolencia e insensibilidad, o como consecuencia de los sistemas de trabajo que les obligan a realizar atenciones inadecuadas y rápidas, violando los derechos de los pacientes, dando prioridad al mercantilismo y a la atención sustentada en la productividad y la comercialización. Actualmente en el Perú existen además razones relacionadas con:

-el excesivo número de facultades de medicina sin acreditación y calidad académica, carentes de personal docente idóneo;

- como consecuencia de lo anterior, la inadecuada formación de cirujanos en sedes sin las condiciones necesarias y sin la acreditación que la ley obliga;

- concentración de los cirujanos en las grandes ciudades y carencia de especialistas en otros lugares del interior del país;

- problemas en los sistemas de salud pública y privada, con sobrecarga de trabajo del médico-cirujano;

-deficiencias en infraestructura y equipamiento en los centros quirúrgicos, que además tienen gran limitación en los petitorios de materiales y medicamentos.

No deberían existir cirujanos deshumanizados e indolentes que menosprecien la esencia espiritual del ser humano y hagan de la codicia lucrativa la razón principal del ejercicio profesional. Ellos habrían perdido la ética y el sentido social y humano de nuestra noble profesión, y es que la tecnología moderna aleja al médico cirujano del paciente, y el sistema de trabajo lo tiene atrapado y lo hace víctima de la burocratización excesiva de los sistemas de salud público y privados.

En la Fundación Instituto Hipólito Unánue, José de Vinatea ${ }^{(32)}$ expresó: “Todos los que trabajamos en las ciencias de la salud estamos vinculados a la dolencia y, por consiguiente, en forma ineludible, a la compasión. Pero esta compasión solo tiene valor en la medida en que esté cobijada del conocimiento, sino se ridiculiza el privilegio de aliviar, se trivializa el sacrificio y se convierten los aciertos en efimeras victorias". Compartimos esta frase porque el cirujano debe fusionar el conocimiento científico con la dedicación, la compasión y el respeto al paciente, recordando la antigua sentencia de los médicos franceses Bérard y Gubler, que 
resumían el papel de la medicina hasta ese momento diciendo: "Curar, pocas veces; aliviar, a menudo; consolar, siempre",(8,9).

En los últimos años hemos sido testigos de grandes avances científicos y tecnológicos en medicina y cirugía. Lamentablemente, éstos no siempre están al alcance de los países en desarrollo, que además viven permanentemente en crisis económicas y tienen una pésima política de salud, lo que deteriora los servicios públicos y trae como consecuencia muchas carencias. Esto ha sido demostrado en la terrible pandemia en la que vivimos actualmente.

Pese a sus grandes beneficios, la tecnología de punta también nos aleja de la medicina y cirugía centrada en la persona. Como decía el maestro Alejandro Villanueva en relación a la inequidad existente en nuestro país: "Ante una enfermedad de tratamiento quirúrgico, el rico se cura y el pobre se muere".

A pesar de lo expuesto, el cirujano es responsable de las situaciones adversas que suceden en un tratamiento quirúrgico y debe justificar y dar cuenta de sus acciones, teniendo en cuenta que a nosotros la vida nos muestra la cara de la muerte y nos enfrenta cotidianamente a ésta.

\section{Ética y Bioética}

La ética apunta al anhelo de realizar el bien, o sea al deber que debemos cumplir para conseguir el bienestar. La bioética significa el comportamiento correcto en relación al ser humano y los conflictos apareados por la instrumentación de la vida del hombre y la naturaleza en sentido amplio ${ }^{(33)}$.

El ejercicio de la cirugía es delicado y riesgoso que obliga a brindar el mejor servicio posible con responsabilidad. El Código de Ética y Deontología del Colegio Médico cumple un importante rol regulador en nuestra práctica profesional y orienta la conducta de los que ejercemos esta profesión hacia el bien, lo correcto, lo ideal y por qué no, hacia la excelencia. Contiene normas de conducta y establece deberes, derechos y valores con los que se debe actuar, enfatizando el estricto respeto a la dignidad, la autonomía e integridad de los pacientes, su familia y la comunidad ${ }^{(34)}$.

El cirujano debe comportarse siempre con decoro, honestidad, desinterés e integridad moral. En el acto quirúrgico se requiere de conocimientos actualizados, adiestramiento, habilidad, destreza y, sobre todo, de acciones responsables para que la cirugía sea segura. Sin embargo, a pesar de lo expresado, la situación actual del cirujano en relación al "paciente quirúrgico" ha sufrido cambios profundos debido a los siguientes factores $^{(35)}$ :
- La bioética, que enlaza los hechos de la vida diaria, la moral y las obligaciones del hombre con las humanidades.

-La socialización de la medicina deteriora la relación médico-paciente, convirtiéndolo en prestador de salud-cliente.

- La promoción de la autonomía del paciente y sus derechos, dentro de los que está el consentimiento informado, libre y consciente en su aceptación y no conminatorio.

-Las exigencias de la socialización y los seguros de salud público y privados.

-El mercantilismo de la atención privada y el marketing opuesto a la ética, que deteriora la imagen del cirujano.

-La "atención gerenciada” que imponen las compañías de seguros particulares, que controlan y limitan los costos de la cirugía.

El cirujano incumple las normas establecidas en las siguientes circunstancias:

-cuando realiza operaciones innecesarias por error diagnóstico, fines de lucro o para recuperar inversiones;

-al operar sin equipos e instrumental adecuados y sin los elementos técnicos indispensables, empleando técnicas operatorias sin la experiencia y la capacidad para realizarlas;

-cuando se omite el consentimiento informado;

-al operar los “casos perdidos", a sabiendas de que no se obtendrá ningún resultado positivo;

- cuando asume posturas de superioridad y soberbia y hace comentarios desmesurados e indebidos sobre los colegas.

Pero no solo son importantes las normas y reglas establecidas, sino los valores morales aprendidos en la universidad de la vida, y la propia conciencia. Literalmente, se trata de cumplir los criterios valorativos de lo correcto: "Lex artis ad hoc ${ }^{,(36,37)}$.

En medicina y cirugía, lex artis ad hoc, en sentido estricto, es tener criterio valorativo de la corrección del acto médico y quirúrgico con prudencia, pericia, diligencia, buena intención y recta conciencia. Es deber ineludible del médicocirujano desempeñar su profesión competentemente, debiendo para ello perfeccionar sus conocimientos, destrezas y de esa manera evitar riesgos y errores que se consideren mala praxis, lo que implica responsabilidad y culpabilidad del cirujano ${ }^{(38,39)}$. 
Se considera mala praxis al resultado de un acto médico y/o quirúrgico que ha producido daño al paciente debido a la aplicación inadecuada de las reglas, métodos, guías, protocolos y procedimientos establecidos. Además, ello implica falla o error del cirujano y afecta su desempeño profesional, lo expone al juzgamiento del Colegio Médico del Perú y a las reclamaciones de los pacientes y sus familiares ante el Poder Judicial, por la vía civil o penal. La mala praxis, excepcionalmente, puede estar relacionada a un inadecuado comportamiento ético y deontológico o, lo que es peor, tener fines subalternos. La mala praxis fortuita constituye un tema discutible pues incorpora, indebidamente, a los eventos adversos, las complicaciones de la enfermedad, la inefectividad de los medicamentos, las reacciones tóxicas y de hipersensibilidad, las infecciones y cuando el resultado final de la cirugía no cumple con el fin para el que fue programada ${ }^{(40)}$.

Los cirujanos estamos obligados a evitar la mala praxis culposa, que es totalmente opuesta a la cirugía centrada en la persona, causa daño y pone en riesgo la vida del enfermo ${ }^{(41,42)}$.

Ésta incluye:

La negligencia, cuando se sale de los estándares, hay falta de cautela y mesura, mala atención, descuido del paciente, delegación de responsabilidades y abandono de éste.

-La impericia, por falta de conocimientos y destreza, inadecuado entrenamiento y deficiente técnica operatoria.

-La imprudencia, por no tener buen juicio y actuar apresuradamente en la realización de un acto quirúrgico innecesario, arriesgado, sin medir sus consecuencias.

- La inobservancia de la normatividad establecida en todos los protocolos.

Por estos hechos, la cirugía se puede convertir en figura penal y puede configurarse como un delito porque el cirujano y su equipo son los responsables del proceso quirúrgico.

\section{Consentimiento informado}

La cirugía desde tiempos remotos ha presentado conflictos éticos que exigen a todo cirujano una elevada formación científica y moral. Para la solución de éstos existen los códigos de ética, cuyas raíces están en el Juramento Hipocrático y las modificaciones hechas por la Asociación Médica Mundial a través del tiempo. Sin embargo, los avances científicos, tecnológicos y bioéticos de los últimos años, han obligado a cambiar la relación vertical entre el cirujano y el paciente, por una relación horizontal y participativa en la que el consentimiento informado tiene un papel muy importante. Es indispensable entender la doble dimensión, la ética y la judicial, de dicho consentimiento, sin confundirlo con una simple autorización para realizar un acto quirúrgico.

En cirugía, el consentimiento informado tiene importancia trascendental porque es la expresión del principio de autonomía del paciente y la aceptación voluntaria de someterse a una cirugía. Se le considera el procedimiento mediante el cual se garantiza que el paciente ha expresado voluntariamente su intención de ser sometido a un tratamiento quirúrgico, después de haber comprendido la información del cirujano sobre los objetivos, beneficios y riesgos de dicho procedimiento. Cumplir con el consentimiento informado es un deber ineludible del cirujano y un derecho del paciente para decidir sobre su salud y su cuerpo. Se le considera como el elemento central de la relación médico-paciente y el resultado de un proceso gradual de información, diálogo y confianza para llegar a decisiones finales en relación al acto quirúrgico ${ }^{(43)}$.

Su historia data del año 1946 con la Fundación de la Asociación Médica Mundial en Londres y el Código de Nurenberg de 1947, que fue el primer documento en el que se planteó el derecho del individuo a dar su consentimiento voluntario para realizar algún acto sobre su cuerpo. En el año 1964, la Asociación Médica Mundial en la Declaración de Helsinki ya se refería al consentimiento voluntario para participar en experimentos con humanos ${ }^{(44)}$.

En el Perú fue instituido y tiene vigencia desde 1997, con la promulgación de la Ley General de Salud, modificada por la Ley 29414 y las demás disposiciones que incluyen una sobre consentimiento informado. También figura dentro de la normas del Código de Ética y Deontología del Colegio Médico del Perú.

El consentimiento informado se basa en los principios de autonomía, beneficencia, solidaridad, justicia y responsabilidad y tiene las siguientes características:

-Transparencia, porque se deja descubrir lo patente de lo declarado;

- Competencia, ya que es atribución legítima del paciente aceptarlo y firmarlo;

-Comprensión, porque el paciente ha sido capaz de entender su contenido;

-Voluntariedad, al aceptarlo y firmarlo por voluntad propia y sin coacción; 
- Respetabilidad, porque las decisiones del paciente son respetadas para salvaguardar las prioridades a su favor.

El consentimiento informado forma parte de la historia clínica y no debe ser un mero asunto protocolar o la firma de un simple formulario que autorice la cirugía. Requiere la obligación imperiosa del cirujano de hacer una explicación minuciosa y clara al paciente y familiares cercanos sobre las alternativas existentes, los beneficios, los riesgos y las posibles complicaciones de la cirugía. Podrá ser revocado por el paciente y servirá para resolver reclamos posteriormente ${ }^{(45,46)}$.

El consentimiento informado debe contener:

-Los nombres, apellidos, número del documento de identidad y huella digital.

-El diagnóstico de la enfermedad.

-El objetivo de la operación y procedimiento a realizarse.

-La preparación pre-operatoria y el tipo de anestesia.

-El curso del post-operatorio.

-Los potenciales riesgos, complicaciones y secuelas.

-Un espacio libre para hacer las anotaciones especiales en cada caso.

-Las firmas del paciente, del testigo y del cirujano.

Por otra parte, el consentimiento informado en cirugía debe cumplir ciertas etapas para ser eficaz:

-Elaboración clara, entendible y asequible a la comprensión del paciente.

-Transmisión al paciente y sus familiares con explicaciones de los pormenores del tratamiento quirúrgico.

-Aceptación del paciente sin ninguna coacción.

- Certificación con la firma del paciente y del familiar más cercano.

Al término del proceso, el consentimiento informado se convierte en un documento importante en el que se garantiza que el paciente ha expresado voluntariamente su aceptación de ser sometido a un tratamiento quirúrgico, después de haber comprendido la información del cirujano, los objetivos, beneficios y riesgos ${ }^{(47)}$.
El consentimiento informado puede ser omitido excepcionalmente en los siguientes casos:

- Cuando existe grave riesgo para la salud pública derivada de una enfermedad infecciosa del paciente.

-En casos de urgencias y/o emergencias que tienen peligro para la vida del paciente y riesgo de muerte, y que requieren de la actuación inmediata del cirujano. Esto se denomina estado de necesidad vital.

•En casos de “privilegio terapéutico”, que le permite al cirujano ocultar información delicada al paciente respecto a un procedimiento quirúrgico de gran envergadura y cuando la revelación supone una grave amenaza para la integridad psicológica del paciente.

Las dificultades que se han encontrado en la elaboración al consentimiento informado son las siguientes:

-El lenguaje técnico y no comprensible para el paciente, y la barrera idiomática.

-Las creencias religiosas que impiden realizar algunos procedimientos como las tranfusiones sanguíneas.

- Cuando el cirujano da una información distorsionada.

-La existencia de formatos desiguales e incompletos y no uniformizados.

Tratándose de una enfermedad oncológica se ha instituido el concepto de "verdad tolerable", para definir el modo adecuado de transmitir la información al paciente y sus familiares, para que puedan soportar la situación de salud grave sin causarles problemas cuando se trata de una enfermedad terminal que conlleva el peligro de muerte. En este caso, el cirujano está obligado a respetar la decisión del paciente ante la negativa de que se realice el acto quirúrgico y/o a la revocatoria del consentimiento informado ya firmado. Ante ésta situación, deberá constar en la historia clínica la explicación y advertencia del cirujano sobre los peligros y riesgos de la no realización de la cirugía programada, de manera que quede a salvo su responsabilidad $^{(48)}$.

Existen situaciones especiales, tratándose de "Grupos Vulnerables". En el caso de menores de edad, por ejemplo, el consentimiento informado debe ser firmado por los representantes legales, padres o tutores. En personas con discapacidad y/o incapacidad, grave trastorno psíquico y deterioro cognoscitivo e inconciencia, el consentimiento informado debe ser otorgado por el curador o un familiar cercano. 
En trasplantes de órganos y tejidos de donantes vivos se debe tener especial cuidado, haciendo la comprobación de que se trata de un acto voluntario y desinteresado del dador. En caso de donantes cadavéricos, el consentimiento es explícito cuando consta en el DNI y presuntivo cuando los deudos aceptan la donación a pesar de no figurar ese deseo en el DNI del cadáver.

\section{Comentario final}

En los últimos años, hemos sido testigos de grandes avances científicos y tecnológicos en medicina y cirugía en los países desarrollados. Dichos avances no conocen fronteras debido a la globalización y a los sistemas de comunicación cada vez más rápidos, pero lamentablemente, no están al alcance de todos, especialmente de los países en desarrollo como el nuestro. En consecuencia, sus beneficios no llegan a la salud de las grandes mayorías por causa de las malas política de salud, la inequidad y la discriminación y, sobre todo, por las grandes diferencias socio-económicas.

A pesar de ello, los médicos y cirujanos estamos obligados a brindar la mejor atención con las limitaciones existentes, tratando de aliviar, curar y dar calidad de vida a nuestros pacientes sin distinción alguna. Insistimos que en el Perú la crisis económica permanente y la mala política de salud afecta a los servicios públicos y ha traído como consecuencia muchas carencias y falta de equidad en la atención de la población más necesitada.

Por otra parte, con la tecnología de punta, nos estamos alejando de la medicina y cirugía centradas en la persona y del humanismo que desde hace siglos enseñaba Sócrates: "Solo un hombre humano puede ser buen médico y buen cirujano". Tratemos de cumplir esa sentencia.

Señalaremos una vez más algunas de las razones por las que el cirujano en nuestro medio tiene limitaciones en su labor cotidiana:

- La deficiente formación académica en pre y post grado en las universidades y la falencia de una educación médicoquirúgica continua.

-Las diferencias socio-económicas y la falta de equidad para acceder a la salud y a los métodos modernos de la cirugía.

-La inadecuada implementación de protocolos y guías de manejo del paciente quirúrgico, convertidos en mordazas que impiden el ejercicio profesional adecuado.

-Las deficiencias en infraestructura, equipamiento e instrumental en los centros quirúrgicos en salud pública.
Para mejorar la calidad del trabajo de los cirujanos en nuestro país sugerimos tomar las siguientes medidas: reducir el número de facultades de medicina no acreditadas, mejorar el nivel académico, el entrenamiento quirúrgico en pre y postgrado a fin de adquirir sólidos conocimientos, habilidades y destrezas; fomentar la educación médica continua, la certificación y la recertificación del Colegio Médico del Perú; equipar adecuada- mente a los centros quirúrgicos, efectuando un estricto control y vigilancia de las salas de operaciones de los hospitales y clínicas; fomentar la programación de intervenciones quirúrgicas con un balance riesgo/beneficio positivo y cumplimiento de los protocolos existentes; exigir la obligatoriedad del consentimiento informado y la estandarización de los procesos para la implementación de la lista de verificación quirúrgica; operar con pleno conocimiento de la responsabilidad legal, civil y penal que obliga a responder por los supuestos perjuicios, balanceando los avances tecnológicos con el humanismo.

La noción de "futilidad terapéutica" en cirugía ocasiona muchas controversias y es motivo de reflexión. Se refiere al límite de las posibilidades de tratamiento frente a la historia natural e inexorable hacia la muerte inminente. En ese caso, cualquier intento de cirugía sería inútil y solo serviría para retrasar la muerte. En este tema el cirujano debe ser consciente y respetar los valores y las creencias religiosas, tratando de resolver el dilema bioético del final de la vida.

A pesar de todo lo expuesto, los cirujanos somos responsables de situaciones adversas, las que debemos justificar y por las que debemos dar cuenta de nuestras acciones, recordando que a nosotros la vida nos muestra la cara de la muerte y nos enfrenta a ella cotidianamente.

No es posible imaginar a un buen cirujano sin cultura humanista, sin vocación de servicio y sin preocuparse por la valoración de los atributos y las relaciones humanas. La cultura humanista es filosofía de vida. Si un cirujano no la posee, es simplemente un técnico que brinda sus conocimientos y habilidades pero no comprende el maravilloso universo del hombre. No debemos olvidar que el respeto a la vida y a la salud constituye la esencia espiritual de nuestro ejercicio profesional y que cualquier acto médico-quirúrgico es una manifestación de la ética para hacer el bien. Ser humanista nos hace entender mejor al paciente, con sus aspiraciones, dudas e interrogantes. Y ser humanitario nos hace más eficientes, bondadosos, comprensivos y generosos.

El cirujano que centra su labor en la persona, enfatiza la ética y la moral, intentando aliviar y curar al enfermo, uniendo la eficacia con la paciencia que da la experiencia. Pero no es 
Dios quien lo puede todo. Es humano, y puede equivocarse. "Errare humanun est". No debemos olvidar que a veces "el cirujano y su equipo tienen en sus manos la vida de sus pacientes"; por ello, el buen cirujano no es el que opera mejor sino el que tiene inteligencia, humildad, altruismo, rigor y caridad pero, sobre todo, cariño a sus pacientes.

Lamentablemente, algunos cirujanos, en su afán de avanzar sin fijarse límites, comienzan a sentirse dioses y en este empeño van perdiendo humanidad y se van alejando del paciente como persona humana.

Debemos seguir sirviendo con amor porque ese es el norte que nos señala la vocación de servicio, y abandonar el paternalismo tratando de conseguir la colaboración del paciente para tomar la decisión respecto a la necesidad de un acto quirúrgico, respetando siempre su autonomía y dignidad.

La cirugía personificada pretende optimizar la cirugía basada en el diagnóstico preciso, considerando al paciente como persona en toda su dimensión humana, lo que hace vigente el viejo aforismo: "No hay enfermedades, sino enfermos".

Los grandes maestros nos enseñaron a unir la observación clínica cuidadosa a la destreza quirúrgica, valorando al paciente como ser humano que sufre y busca su curación. Nuestros pacientes nos van a estimar, no solo por nuestra habilidad quirúrgica sino por nuestra bondad. Cuando se pierde al paciente debemos recordar que "la vida no es para siempre y la muerte es un hecho ineludible”.

Un mensaje final: "La libertad de las personas y el respeto al prójimo son las bases sólidas que todo cirujano debe forjar en su historia profesional. Sobre ellas, construirá su mundo, con la confianza suficiente en su pasado, para seguir creciendo y forjando un futuro mejor".

\section{Referencias bibliográficas}

1. World Health Organization. Rev. World Health Assembly, 62. 16. 2009. Genova. Italia.

2. Pacheco OL. La Cirugía Centrada en la Persona. Facultad de Ciencias Médicas. Quito, 2018;43(1):7-10.

3. Alvarez M. Nuevo Paradigma: Medicina Centrada en la Persona. Rev. Bioética y Ciencias de la Salud. Andalucía. 2019;7:2.

4. Wagner $P$, et al. Bases y Perspectivas Latinoamericanas sobre Medicina y Salud Centrada en la Persona, Anales Facultad de Medicina, 2015;76(1).

5. Espinoza A. La Medicina Centrada en la Persona, Rev. Med. Sur, Cuba. 2015;13(6).

6. León Barúa R. Medicina Centrada en la Persona: Perspectivas Clínicas. Revista Médica Herediana, 2010;21(3).

7. Mezzich JE. Repensando el Centro de la Medicina: De la Enfermedad a la Persona. Acta Med. Per. 2010;27(2):112-119.

8. Haro DE. La Medicina, la Cirugía y la Oftalmología en la antigüedad. Para una Historia en la Oftalmología. Edición Forma \& Imagen, Lima. 2008:19-32.

9. Lain P. Historia de la Medicina. Editorial Masson 1a. Edición. España. 1978

10. Bueso A. El Juramento Médico. Rev. Med, Honduras.2004;72 (2):114-115.

11. Bonds C. Hipocrattic Oath: A bassics for modern ethical standars. JAMA. 1990;264:2311.

12. Lasagna L. Modern Version Medical Oath. Univer. California. Ethic Center 2013:9-21.

13. Biblia Católica. MCMLXV. Evangelio de San Lucas. 10:2732. Stamply, 1950.

14. Druker B. Ambroise Pare and the birth of gentle art of surgery.
Yale Journal of Biology and Medicine, 2008.

15. Garcia, D. Bioética Clínica, Editorial El Búho 1era. Edición, Bogotá- Colombia, 1998.

16. American Board of Internal Medicine. American College Of Physicians. European Federation of Internal Medicine. Profesionalismo Médico en el Nuevo Milenio. An. Inter. Med. Bulletin of American College of Surgeons. 1996;68:10.

17.Declaración de Alma-Ata. http.//www.org/spanish/dd/pin alma -ata declaración. htm

18.Baracco V. Fundación de la Academia Peruana de Cirugía. Revista APC. 2000;49(2).

19.Vidal J. Visión Histórica de los Forjadores de la Cirugía en el Perú. Rev Especializadas Peruanas. Lima. 2008.

20.Haro DE. Origen de la Academia Peruana de Cirugía. Rev. Academ Peruana Cir. 2010;1(3):43-45.

21.Real Academia Española. Diccionario de la Lengua Española XIX. Edición 1970. Espasa \& Calpe S.A. Madrid.

22.Anaya-Prado R, et. al. Praxis Médica. Rev. Cir. Gen. Guadalajara-Mexico. 2013;35(2):164-166.

23. Ramirez A. La Faceta Humana del Médico. Rev. Diagnóstico (FIHU) 1995;34(2);36-42.

24.Nizama VM. Humanismo Médico. Anales Facultad de Medicina, 2001;15(1). Disponible enhttp//sisbib.unmsm. edu.pe.

25.Delgado H. El Médico, la Medicina y el Alma. Ediciones UPCH, Lima. 1992.

26.Seguín CA. La Enfermedad, el Enfermo y el Médico. Ediciones Pirámide S.A. Madrid. 1982.

27.Mendoza M. Reflexiones sobre Humanismo y Medicina. Acta Médica Colombiana, 1998;23(6):130-133. 
28. Mesa-Piñas A. La Humanización de la Medicina en el Siglo XXI. Ed, CVU. Madrid. 2016.

29.Pérez TR. Humanismo y Medicina. Gaceta Médica de México. 2013;149:349-353.

30.Academia Nacional de Medicina. Declaración de Lima. Educación Médica Centrada en la Persona. Rev. Diagnóstico (FIHU). Lima. 2019;58(1)48-49.

31. Mezzich JE. Educación Médica Centrada en la Persona. Rev. Diagnóstico (FIHU). 2019;56(1):33-39.

32. De Vinatea J. Discurso Fundación Instituto Hipòlito Unánue, Rev. Diagnóstico (FIHU). 2018;55(4):215-217.

33. Gracia D. Fundamentos de Bioética. Ed. Tracastela, Segunda Edición Madrid Vigentes. 2008.

34. Colegio Médico del Perú. Normas y disposiciones Legales Vigentes. Código de Ética y Deontología. Lima. 2018.

35. Arroyo, F. Reflexiones Éticas en la Práctica de la Cirugía. Rev. Chilena de Cirugía, 2008;60(4):352-356.

36. Gurmendi, L. Ética en Cirugía. Rev. Academia Peruana de Cirugía, Perú. 1982;30:1-2.

Zaldívar C. Ética en Cirugía. Principios y Terapéutica Quirúrgica. Barboza Didi de Arteta S.A 1 Edición, 1999: 90.

38. Lerma C. Dilemas Eticos del Cirujano, Rev. Colombiana de Cirugía, 2000;15(4):222-225. https//encolombia.com/ medicina/revistas-medicas/cirugía/vc-154/cirugia15400

39. Moore FD. Ethical Problems Special to Surgery. Arch Surg 2000:135:14-16

40. Haro DE. Ética y Deontología en Cirugía y Oftalmología. Para una Historia de la Oftalmología en el Perú. Ed. Forma e Imagen. 2008;173-179.

41. Helton CR. Ethics in Surgery. J. Am. Coll. Surg. 1986:1.

42. Gutiérrez W. Los Derechos de los Pacientes. Rev. Per. Soc. Med. Inter. Lima. 2014;27(1):33-39.

43. Fernández SC. La Responsabilidad Civil del Médico y el Consentimiento Informado. Ed. Montieva. Lima. 2011.

44. Asociación Médica Mundial. Declaración de Helsinki. Principios Éticos para las Investigaciones Médicas en Seres Humanos, $1964 \mathrm{https} / /$ www.um.es $<$ documents.

45. Cárdenas D. El Consentimiento Informado y la Responsabilidad Médica. Col Der. Priv. Imprenta Nacional de la Agencia Estatal. Madrid. 2018.

46. Galán C. El Consentimiento Informado en la Práctica Médica. Ed. Colex. Madrid. 1997.

47. López FD. Aspectos Legales y Éticos del Consentimiento Informado. Rev. Mex. Patol. Clin. 2001;48(1):3.

48. Burdile J. Consentimiento Informado en Cirugía. Rev. Chil. Cir. 2004;58(2):97-98.

Contribución de autoría: Daniel Enrique Haro-Haro ha sido el autor del artículo, contribuyendo con su concepción, recopilación de información, redacción, revisión y edición final. Augusto B. Chafloque-Cervantes ha sido el autor del artículo, contribuyendo con su concepción, recopilación de información, redacción, revisión y edición final.

Conflicto de interés: Los autores no tienen conflictos de interés con la publicación de este trabajo.

Financiamiento: Autofinanciado.

Citar como: Haro Haro DE, y col. Cirugía Centrada en la Persona. Diagnóstico(Lima). 2021;60(1):50-60.

DOI: 10.33734/diagnostico.v60i1.272

Correspondencia: Daniel Enrique Haro Haro. Correo electrónico: haroharodanielenrique@gmail.com

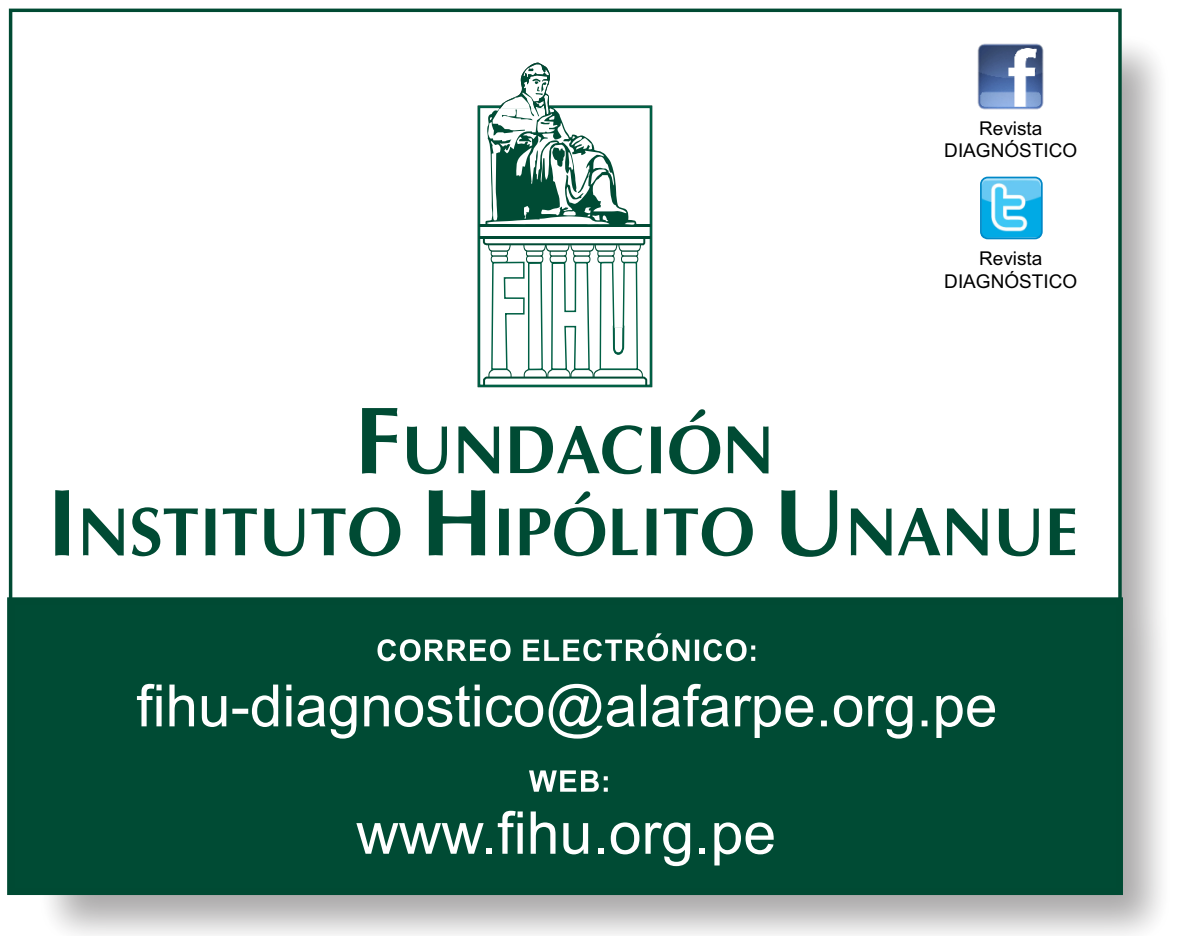

